\title{
Monodromies and the structure of gauge and gravity amplitudes
}

\author{
Pierre Vanhove ${ }^{* \dagger}$ \\ Institut des Hautes Etudes Scientifiques \\ Le-Bois-Marie \\ F-91440 Bures-sur-Yvette, France \\ and \\ Institut de Physique Théorique, \\ CEA, IPhT, F-91191 Gif-sur-Yvette, France \\ CNRS, URA 2306, F-91191 Gif-sur-Yvette, France \\ E-mail: pierre.vanhove@cea.fr
}

\begin{abstract}
Although systematic the Feynman graph approach to perturbative calculations in gauge and gravity is far from being optimal. Combining insights from string theory and on-shell unitarity methods, new approaches have been designed. In the case of maximally supersymmetric theories, they have led to the discovery of unsuspected similarities in the structure of perturbative gauge theory and supergravity. We will present these new approaches and discuss the consequences on ultraviolet question of maximal supergravity.
\end{abstract}

35th International Conference of High Energy Physics

July 22-28, 2010

Paris, France

*Speaker.

${ }^{\dagger}$ IPHT-T11/013. 


\section{Tree-level amplitudes}

Close to a century after its first formulation, the general theory of relativity continues to give us surprises. In particular, remarkable new insight arises from the intimate relation between gravity and Yang-Mills theories. In perturbation theory one of the most intriguing manifestations of this comes from the factorization of closed string amplitudes into products of open string amplitudes, the Kawai-Lewellen-Tye (KLT) relations [1]. In the field theory limit, these relations show that multi-graviton tree-level amplitudes can be expressed as products of color-ordered Yang-Mills amplitudes.

These KLT-relations between gravity amplitudes and Yang-Mills amplitudes have been proven in a series of different but equivalent forms using only concepts from relativistic quantum field theory using on-shell unitarity method in [?,2,3] and string theory $[1,4,5]$.

From these constructions emerge as central object a momentum kernel $[?, 3,5]$

$$
\mathscr{S}_{\alpha^{\prime}}\left[i_{1}, \ldots, i_{k} \mid j_{1}, \ldots, j_{k}\right]_{p} \equiv\left(\pi \alpha^{\prime} / 2\right)^{-k} \prod_{t=1}^{k} \sin \left(\pi \alpha^{\prime}\left(p \cdot k_{i_{t}}+\sum_{q>t}^{k} \theta\left(i_{t}, i_{q}\right) k_{i_{t}} \cdot k_{i_{q}}\right)\right),
$$

where $\theta\left(i_{t}, i_{q}\right)$ equals 1 if the ordering of the legs $i_{t}$ and $i_{q}$ is opposite in the sets $\left\{i_{1}, \ldots, i_{k}\right\}$ and $\left\{j_{1}, \ldots, j_{k}\right\}$, and 0 if the ordering is the same.

This momentum kernel maps products of open string amplitudes to closed string amplitudes and allows to express the closed string amplitudes as

$$
\begin{aligned}
& \mathfrak{M}_{n}=(-i / 4)^{n-3} \times \\
& \sum_{\sigma} \sum_{\gamma, \beta} \mathscr{S}_{\alpha^{\prime}}[\gamma(\sigma(2), \ldots, \sigma(j-1)) \mid \sigma(2, \ldots, j-1)]_{k_{1}} \mathscr{S}_{\alpha^{\prime}}[\beta(\sigma(j), \ldots, \sigma(n-2)) \mid \sigma(j, \ldots, n-2)]_{k_{n-1}} \\
& \quad \times \quad \mathscr{A}_{n}(1, \sigma(2, \ldots, n-2), n-1, n) \widetilde{\mathscr{A}_{n}}(\gamma(\sigma(2), \ldots, \sigma(j-1)), 1, n-1, \beta(\sigma(j), \ldots, \sigma(n-2)), n) .
\end{aligned}
$$

where the sum is over the permutations $\sigma$ of $n-3$ external states, $\gamma$ and $\beta$ of $j-2$ external states. This expression is independent of choice of $j$ between 3 and $n-1$. In the field theory limit $\alpha^{\prime} \rightarrow 0$ the expression in (1.1) turns into the field theory momentum kernel $\mathscr{S}$ constructed in [3].

Starting from closed type II string, the $\alpha^{\prime} \rightarrow 0$ limit gives the expression for multi-graviton tree-level amplitude expressed in terms of color-stripped multi-gluon amplitudes. Starting from the heterotic string one can derive the expression for multi-gluon tree-level amplitude from colorstripped multi-gluon amplitudes and adjoint scalar amplitudes.

In beautiful agreement with the corresponding observation in field theory, the string theory momentum kernel $\mathscr{S}_{\alpha^{\prime}}$ is precisely the generator of monodromy relations satisfied by the colorordered gauge theory amplitudes [6-8,11]. It annihilates color-ordered amplitudes $\mathscr{A}_{n}$ according to

$$
\sum_{\sigma \in \mathfrak{S}_{n-2}} \mathscr{S}_{\alpha^{\prime}}[\sigma(2, \ldots, n-1) \mid \beta(2, \ldots, n-1)]_{k_{1}} \mathscr{A}_{n}(n, \sigma(2, \ldots, n-1), 1)=0
$$


where $\beta$ is any permutation of leg $2, \ldots, n-1$ and the sum runs over all permutations of these legs. The resulting linear system is non-degenerate for a generic choice of momenta and can be inverted to express the color-ordered amplitudes in the minimal basis of $(n-3)$ ! amplitudes $\mathscr{A}_{n}(n, \sigma(2, \ldots, n-2), n-1,1)$ with $\sigma$ a permutation of $n-3$ external states [6].

For the choice of $j=n-1$ the amplitude in (1.2) takes the nice form given by the expansion of the gravity and gauge theory amplitudes on the minimal basis

$$
\begin{aligned}
\mathfrak{M}_{n}=(-1)^{n-3} \sum_{\sigma, \gamma} \mathscr{S}_{\alpha^{\prime}}[\gamma(2, \ldots, n-2) \mid \sigma(2, \ldots, n-2)]_{k_{1}} \\
\quad \times \mathscr{\mathscr { A }}_{n}(1, \sigma(2, \ldots, n-2), n-1, n) \widetilde{\mathscr{A}_{n}}(n-1, n, \gamma(2, \ldots, n-2), 1),
\end{aligned}
$$

where $\sigma$ and $\gamma$ are permutations of $n-3$ external legs.

This momentum kernel formalism allows to derive various interesting properties for tree-level amplitudes, like new non-linear relations among Yang-Mills amplitudes [10] that can be understood in the context of $R$-charges conservation [11-13].

\section{Dressing cubic graphs}

So far we have discussed generic properties of the tree-level multiparticle amplitudes in gauge and gravity without resorting to an explicit parametrisation.

A parametrisation based on dressed $\varphi^{3}$ graphs has been introduced by Bern, Carrasco and Johansson at tree-level in [14] and generalized at loop orders in [15].

Tree-level gauge and gravity $n$-point amplitudes can be represented as

$$
\mathfrak{A}_{n}^{\text {tree }}=\sum_{\gamma \in \Gamma_{n}^{\text {rree }}} \frac{n_{\gamma} c_{\gamma}}{\prod_{\alpha \in \gamma} p_{\alpha}^{2}} ; \quad \mathfrak{M}_{n}^{\text {tree }}=\sum_{\gamma \in \Gamma_{n}^{\text {tree }}} \frac{n_{\gamma} \tilde{n}_{\gamma}}{\prod_{\alpha \in \gamma} p_{\alpha}^{2}}
$$

where the sums now run over all distinct $n$-point tree-graphs $\Gamma_{n}^{\text {tree }}$ constructed with cubic vertices only. The inverse propagators of the diagram are $p_{r}^{2}, c_{\gamma}$ are color factors, and $n_{\gamma}$ are Lorentz factors. The color factors are obtained by assigning to each (three-point) vertices a structure constant $f_{b c}^{a}=$ $-f_{c b}^{a}$ and to each propagator a Kronecker delta $\delta_{a b}$ where $a, b, c$ are indices in the fundamental representation of the gauge group $G$. Because the structure constants satisfy the Jacobi identity $f_{a b}^{d} f_{c d}^{e}+f_{c a}^{d} f_{b d}^{e}+f_{b c}^{d} f_{a d}^{e}=0$, the color factors $c_{i}$ associated to set of graphs differing only by the permutation of three external legs satisfy the Jacobi relation $c_{i}+c_{j}+c_{k}=0$.

The Lorentz factor $n_{\gamma}$ have ambiguities corresponding to the freedom in reabsorbing higherpoint vertices. In gauge theory only four-point vertices need to be re-expressed by inserting factors $p_{\alpha}^{2} / p_{\alpha}^{2}=1$. In gravity theory one needs to reabsorb all higher-point vertices. The individual Lorentz factors do not have to be gauge invariant. A consistency condition is that these factors satisfy a Jacobi-like relations $n_{i}+n_{j}+n_{k}=0$ similar to the one satisfied by the color factors $[?, 14]$. Such relations, that are necessary for a correct parametrisation the amplitudes, where shown in [7] to be a consequence of the monodromy relations discussed in section 1. This derivation indicates that the Lorentz numerator factors satisfy a generalized Jacobi-like identity $n_{i}+n_{j}+n_{k}=P_{n}\left(k_{r} \cdot k_{s}\right)$. The right-hand side reflects the ambiguity in reabsorbing the higher-point vertices into three-point vertices. 
As indicated in (2.1), the numerators of gravity amplitudes are given by two copies of the Lorentz factors. Only one family of numerator needs to satisfy the strict (without right-hand-side, $P_{n}=0$ ) Jacobi-like relations $[7,15]$. In the case both numerator factors satisfy a generalized Jacobirelation the right-hand-sides need to satisfy a consistency relation. A five-point example is given in [7, section 5].

Such a parametrisation has been generalized at loop orders in $[14,15]$. The $L$-loop four-point amplitudes in $\mathscr{N}=4$ super-Yang-Mills and $\mathscr{N}=8$ supergravity take the form [15]

$$
\mathfrak{A}_{n, L}^{(D)}=g_{\mathrm{YM}}^{2 L+2} \sum_{j} \int \prod_{i=1}^{L} \frac{d^{D} \ell_{i}}{(2 \pi)^{D}} \frac{1}{S_{j}} \frac{n_{j} c_{j}}{\prod_{r=1}^{3 L+1} p_{r}^{2}} ; \quad \mathfrak{M}_{n, L}^{(D)}=\kappa_{(D)}^{2 L+2} \sum_{j} \int \prod_{i=1}^{L} \frac{d^{D} \ell_{i}}{(2 \pi)^{D}} \frac{1}{S_{j}} \frac{n_{j} \tilde{n}_{j}}{\prod_{r=1}^{3 L+1} p_{r}^{2}}
$$

where $S_{j}$ are symmetry factors of the corresponding Feynman diagrams. This parametrisation requires to include 1-particle reducible graphs arising contact terms [15-17]. The supergravity amplitudes are build from two copies of the super-Yang-Mills Lorentz numerators. The constraints on the Lorentz numerators factor are similar to the one at tree-level order [15].

The pure spinor formalism provides a consistent approach to this $\varphi^{3}$ graph parametrisation of $\mathscr{N}=4$ super-Yang-Mills and $\mathscr{N}=8$ supergravity amplitudes at tree-level [18] and loop order [17].

\section{Superficial ultraviolet behaviour of $\mathscr{N}=8$ amplitudes}

The question of the ultraviolet behaviour of a supersymmetric theory requires a very good control of supersymmetry in perturbation. In general only part (half) of the supersymmetry is explicitly realized and the action of the rest of the supersymmetry transformations can be difficult to analyze.

The particular parametrisation of the multiloop amplitude in $\mathscr{N}=4$ super-Yang-Mills and $\mathscr{N}=8$ supergravity in (2.2) indicates possible relations between the ultraviolet behaviour of the two theories.

The leading ultraviolet behaviour of the four-point $L$-loop amplitude in $\mathscr{N}=4$ super-YangMills is given by [16,19-21]

$$
D=8 \quad \text { for } \quad L=1 ; \quad D \geq 4+\frac{6}{L} \quad \text { for } \quad L \geq 2 .
$$

This critical dimension reflects that the leading low-energy behaviour of the amplitude- $t_{8} \operatorname{tr} F^{4} \Lambda^{D-8}$ at one-loop and $\partial^{2} t_{8} \operatorname{tr}\left(F^{4}\right) \Lambda^{(D-4) L-6}$ for $L \geq 2$-arises from the planar sector. The leading ultraviolet behaviour in the non-planar sector is given by $[16,17,21]$

$$
D \geq 4+\frac{4+2\lceil L / 2\rceil}{L} \quad \text { for } L \leq 4 ; \quad D \geq 4+\frac{8}{L} \quad \text { for } L \geq 4 .
$$

This critical dimension reflects that the leading low-energy behaviour in the non-planar sector of the amplitude is given by $t_{8}\left(\operatorname{tr} F^{2}\right)^{2} \Lambda^{D-8}$ at one-loop, $\partial^{2} t_{8}\left(\operatorname{tr} F^{2}\right)^{2} \Lambda^{2(D-7)}$ at two-loop and $\partial^{4} t_{8}\left(\operatorname{tr} F^{2}\right)^{2} \Lambda^{(D-4) L-8}$ from three-loop order [16,17]. 
Since the four-point $\mathscr{N}=8$ supergravity is build from two copies of the $\mathscr{N}=4$ super-YangMills Lorentz numerators factors, one concludes [22] that the worst ultraviolet of the four-point amplitudes in $\mathscr{N}=8$ behaviour is given by

$$
D \geq 4+\frac{6}{L} \quad \text { for } L \leq 4 ; \quad D \geq 2+\frac{14}{L} \quad \text { for } L \geq 4
$$

Such behaviour has been supported by a world-line pure spinor analysis in [17].

The rule (3.3) implies that the $L=5$ loop four-point amplitude in $\mathscr{N}=8$ supergravity is ultraviolet divergent whereas the the $\mathscr{N}=4$ super-Yang-Mills is finite.

In four dimensions the rule (3.3) implies a logarithmic ultraviolet divergence at seven-loop order in the four-graviton amplitude. A candidate counterterm is the dimension sixteen operator $\partial^{8} t_{8} t_{8} \mathscr{R}^{4}$ given by eight derivative distributed on four powers of the Riemann tensor. Such operator have been shown to be compatible with the $E_{7}$ symmetry of $\mathscr{N}=8$ supergravity in [23,24]. The volume of superspace could provide an $E_{7} \mathscr{N}=8$ supersymmetric representation for this counterterm

$$
\int d^{4} x \sqrt{-g^{(4)}}\left(\partial^{8} t_{8} t_{8} \mathscr{R}^{4}+\cdots\right) \sim \int d^{4} x \int d^{32} \theta|E|,
$$

where $|E|$ is the determinant of the super-vierbein. To the contrary to the volumes of $\mathscr{N}=1$ and $\mathscr{N}=2$ superspace this expression does not seem to be vanishing.

\section{Acknowledgements}

I would like to thank N. Berkovits, N.E.J. Bjerrum-Bohr, P.H. Damgaard, M.B. Green, S. Miller, J. Russo, and T. Søndergaard for collaborations on the work reported here.

\section{References}

[1] H. Kawai, D. C. Lewellen and S. H. H. Tye, Nucl. Phys. B 269 (1986) 1.

[2] Z. Bern, L. J. Dixon, M. Perelstein and J. S. Rozowsky, Nucl. Phys. B 546 (1999) 423 [hep-th/9811140].

[3] N. E. J. Bjerrum-Bohr, P. H. Damgaard, B. Feng and T. Sondergaard, Phys. Rev. D 82 (2010) 107702 [arXiv:1005.4367 [hep-th]]. N. E. J. Bjerrum-Bohr, P. H. Damgaard, B. Feng and T. Sondergaard, JHEP 1009 (2010) 067 [1007.3111 [hep-th]]. B. Feng, R. Huang and Y. Jia, Phys. Lett. B 695 (2011) 350 [arXiv:1004.3417 [hep-th]]. Y. X. Chen, Y. J. Du and B. Feng, arXiv:1101.0009 [hep-th]. Y. Jia, R. Huang and C. Y. Liu, Phys. Rev. D 82 (2010) 065001 [arXiv:1005.1821 [hep-th]]. B. Feng, S. He, R. Huang and Y. Jia, JHEP 1010 (2010) 109 [arXiv:1008.1626 [hep-th]]. D. Vaman and Y. P. Yao, JHEP 1011 (2010) 028 [arXiv:1007.3475 [hep-th]]. T. Sondergaard, Nucl. Phys. B 821 (2009) 417 [0903.5453 [hep-th]].

[4] Z. Bern, A. De Freitas and H. L. Wong, Phys. Rev. Lett. 84 (2000) 3531 [hep-th/9912033].

[5] N. E. J. Bjerrum-Bohr, P. H. Damgaard, T. Sondergaard and P. Vanhove, JHEP 1101 (2011) 001 [arXiv:1010.3933 [hep-th]].

[6] N. E. J. Bjerrum-Bohr, P. H. Damgaard and P. Vanhove, Phys. Rev. Lett. 103 (2009) 161602 [arXiv:0907.1425 [hep-th]]. 
[7] N. E. J. Bjerrum-Bohr, P. H. Damgaard, T. Sondergaard and P. Vanhove, JHEP 1006 (2010) 003 [1003.2403 [hep-th]].

[8] S. Stieberger, arXiv:0907.2211 [hep-th].

[9] S. H. Henry Tye and Y. Zhang, JHEP 1006 (2010) 071 [arXiv:1003.1732 [hep-th]].

[10] N. E. J. Bjerrum-Bohr, P. H. Damgaard, B. Feng and T. Sondergaard, Identities among Gauge Theory Amplitudes," Phys. Lett. B 691 (2010) 268 [1006.3214 [hep-th]].

[11] H. Tye and Y. Zhang, 1007.0597 [hep-th].

[12] B. Feng and S. He, JHEP 1009 (2010) 043 [1007.0055 [hep-th]].

[13] H. Elvang and M. Kiermaier, JHEP 1010 (2010) 108 [arXiv:1007.4813 [hep-th]].

[14] Z. Bern, J. J. M. Carrasco and H. Johansson, Phys. Rev. D 78 (2008) 085011 [0805.3993 [hep-ph]].

[15] Z. Bern, J. J. M. Carrasco and H. Johansson, Phys. Rev. Lett. 105 (2010) 061602 [arXiv:1004.0476 [hep-th]]. Z. Bern, T. Dennen, Y. t. Huang and M. Kiermaier, Phys. Rev. D 82 (2010) 065003 [arXiv:1004.0693 [hep-th]].

[16] N. Berkovits, M. B. Green, J. G. Russo and P. Vanhove, JHEP 0911 (2009) 063 [arXiv:0908.1923 [hep-th]].

[17] J. Bjornsson and M. B. Green, JHEP 1008 (2010) 132 [arXiv:1004.2692 [hep-th]]. J. Bjornsson, JHEP 1101 (2011) 002 [arXiv:1009.5906 [hep-th]].

[18] C. R. Mafra, JHEP 1001 (2010) 007 [arXiv:0909.5206 [hep-th]]. C. R. Mafra, O. Schlotterer, S. Stieberger and D. Tsimpis, arXiv:1012.3981 [hep-th]. C. R. Mafra, O. Schlotterer, S. Stieberger and D. Tsimpis, arXiv:1011.0994 [hep-th]. C. R. Mafra, JHEP 1011 (2010) 096 [arXiv:1007.3639 [hep-th]].

[19] Z. Bern, L. J. Dixon, D. C. Dunbar, M. Perelstein and J. S. Rozowsky, Nucl. Phys. B 530 (1998) 401 [arXiv:hep-th/9802162].

[20] Z. Bern, J. J. M. Carrasco, L. J. Dixon, H. Johansson and R. Roiban, Phys. Rev. D 78 (2008) 105019 [arXiv:0808.4112 [hep-th]].

[21] Z. Bern, J. J. M. Carrasco, L. J. Dixon, H. Johansson and R. Roiban, Phys. Rev. D 82 (2010) 125040 [arXiv:1008.3327 [hep-th]].

[22] P. Vanhove, arXiv:1004.1392 [hep-th].

[23] H. Elvang, D. Z. Freedman and M. Kiermaier, JHEP 1011 (2010) 016 [arXiv:1003.5018 [hep-th]]. H. Elvang, D. Z. Freedman and M. Kiermaier, arXiv:1012.3401 [hep-th]. N. Beisert, H. Elvang, D. Z. Freedman, M. Kiermaier, A. Morales and S. Stieberger, Phys. Lett. B 694 (2010) 265 [arXiv:1009.1643 [hep-th]]. D. Z. Freedman and E. Tonni, arXiv:1101.1672 [hep-th].

[24] G. Bossard, P. S. Howe and K. S. Stelle, Phys. Lett. B 682 (2009) 137 [arXiv:0908.3883 [hep-th]]. G. Bossard, C. Hillmann and H. Nicolai, JHEP 1012 (2010) 052 [arXiv:1007.5472 [hep-th]]. G. Bossard, P. S. Howe and K. S. Stelle, JHEP 1101 (2011) 020 [arXiv:1009.0743 [hep-th]]. 